Supplement to the Annals of Applied Statistics

\title{
BAYESIAN HIERARCHICAL RULE MODELING FOR PREDICTING MEDICAL CONDITIONS
}

\author{
By Tyler H. McCormick* ${ }^{*}$ Cynthia Rudin ${ }^{\dagger}$ And David Madigan ${ }^{\ddagger}$ \\ University of Washington*, Massachusetts Institute of Technology ${ }^{\dagger}$ and \\ Columbia University ${ }^{\ddagger}$
}

\section{SUPPLEMENTARY MATERIAL}

\section{Supplement A: Additional simulation results}

In the main text, we consider a combination of popular and randomly selected rules. In Figure 1, we present results for a similar simulation setup with the top 50 most common rules. While HARM still outperforms the other methods we consider, the distinction is less than with the randomly selected rules. This difference reflects the benefits of the hierarchical structure in HARM for rare rules.

The second set of supplementary material concerns model selection. In the text, we use a model which contains all of the available covariates. Using this model highlights the flexibility of our approach, but is not necessarily the model which will give the best predictions. We fit a series of models with an intercept and one demographic factor (age, race, gender, or treatment). We also fit a model with only an intercept term. Figures 2 and 3 present boxplots of the predictive performance of several alternative models. The overall out-of-sample performance, generated by combining new and partially observed patients, is displayed in Figure 4. The out-of-sample performance of the more complicated model (the full model) is not as good as the out-of-sample performance on some of the simpler models with fewer covariates. Since this often indicates a problem with overfitting, we looked at the in-sample performance to see whether it agreed with the out-of-sample performance. Figure 5 presents boxplots of the in-sample predictive performance of the models. As it turns out, the in-sample and out-of-sample performance were similar, so there is no problem with generalization. To determine why the simpler models performed better in-sample, we note that the evaluation metric for the algorithm's performance is different from the

AMS 2000 subject classifications: Primary 60K35, 60K35; secondary 60K35

Keywords and phrases: Association rule mining, healthcare surveillance, hierarchical model, machine learning 

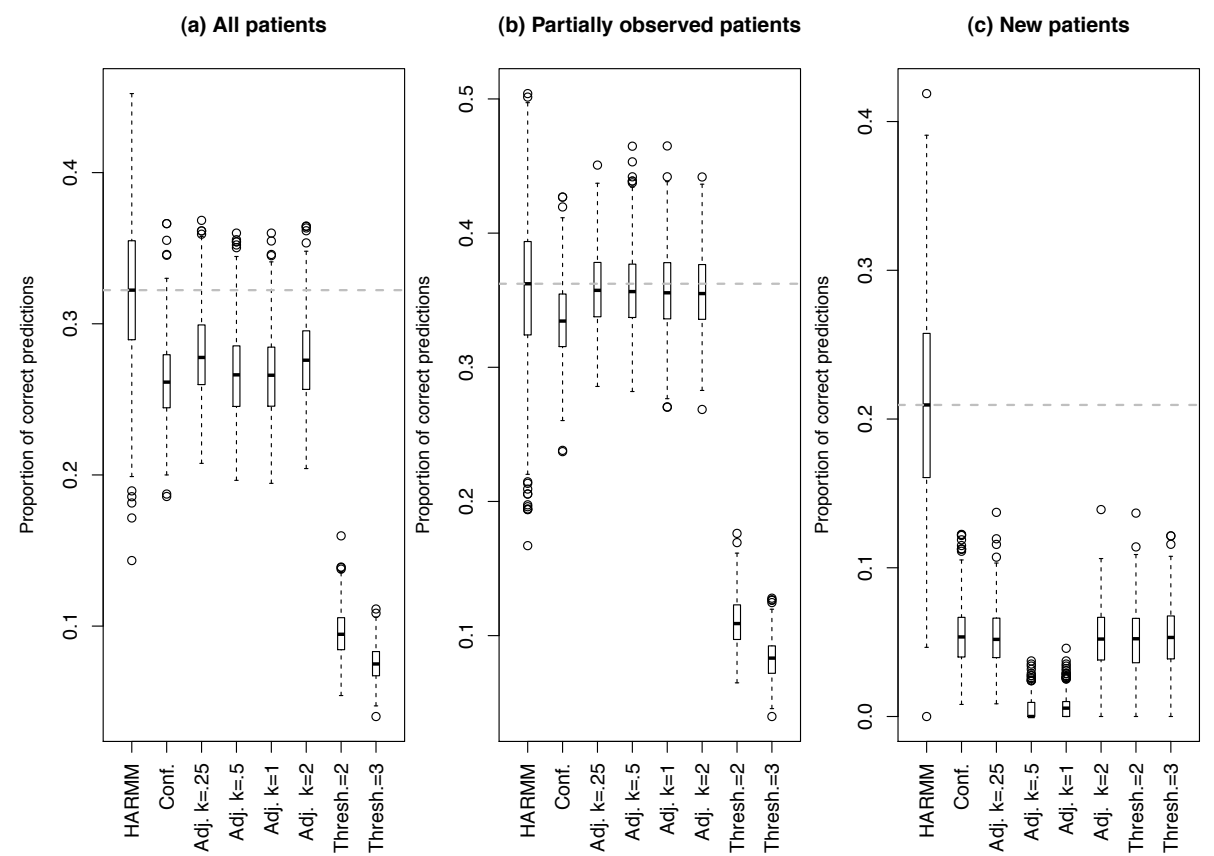

FIG 1. Simulation experiment with common rules. Each boxplot represents the performance of HARM and other methods for 500 simulation iterations. In each simulation, 200 patients are randomly selected and evaluated using the 50 most common rules.

quantity it actually optimizes. This means we are not directly optimizing performance, and this mismatch could potentially cause a substantial difference in performance between different algorithms.

In practice, we suggest additional model selection in cases where prediction is the main goal. The results we present in Figures 2, 3, and 4 could be the conceived as the beginning of a forward stagewise procedure based on the simulation strategy outlined in the paper.

In many cases, the best subset for prediction may change over time. In such cases the computationally intensive strategies we have proposed will likely not be pragmatic. Instead, an approach based on, for instance, Bayes Factors or one-step-ahead prediction could be implemented.

AdDress OF THE First aUthor

Department of Statistics UNIVERSITY OF WASHINGTON

Box 354320 Seattle, WA 98105, USA

E-MAIL: tylermc@u.washington.edu
Address of the Second author

Mit Sloan School of Management, E62-576 Massachusetts Institute of Technology

Cambridge, MA 02139, USA

E-MAIL: rudin@mit.edu 


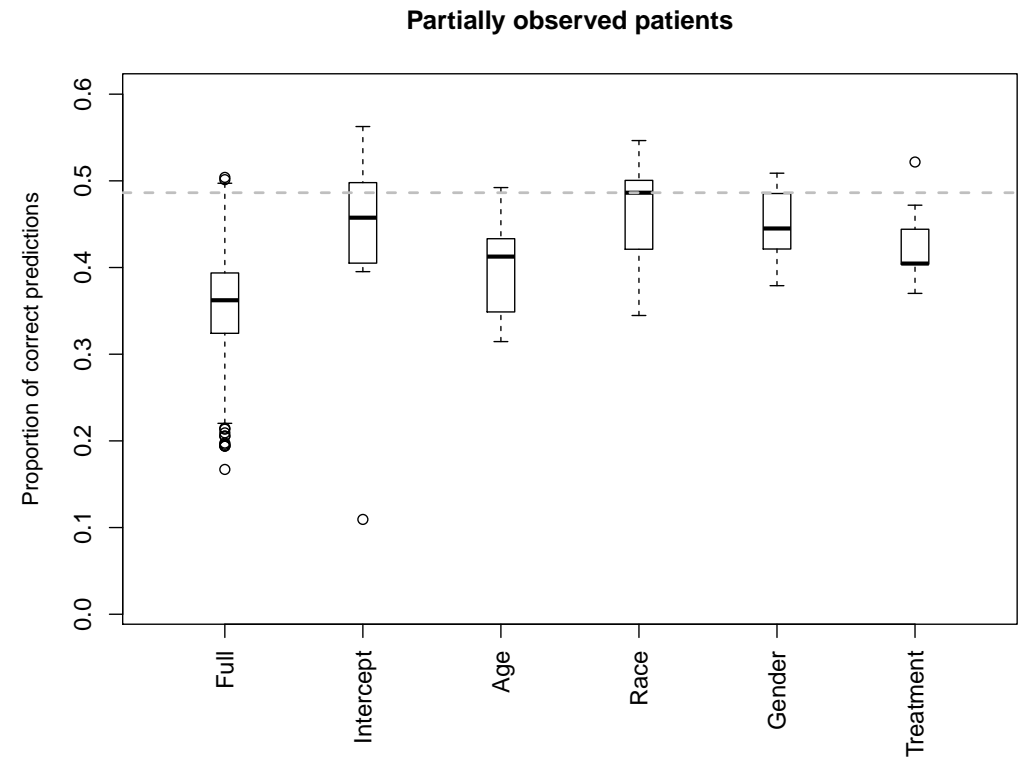

FIG 2. Simulation experiment for model selection using partially observed patients. Each boxplot represents the performance of HARM with a subset of the available covariates. The model using just race covariates performed the best out of the models we considered.

AdDREsS OF THE THIRD AUTHOR

DePARTMENT OF Statistics

COLUMBia University

1255 Amsterdam Ave. New York, NY 10027, USA

E-MAIL: madigan@stat.columbia.edu 


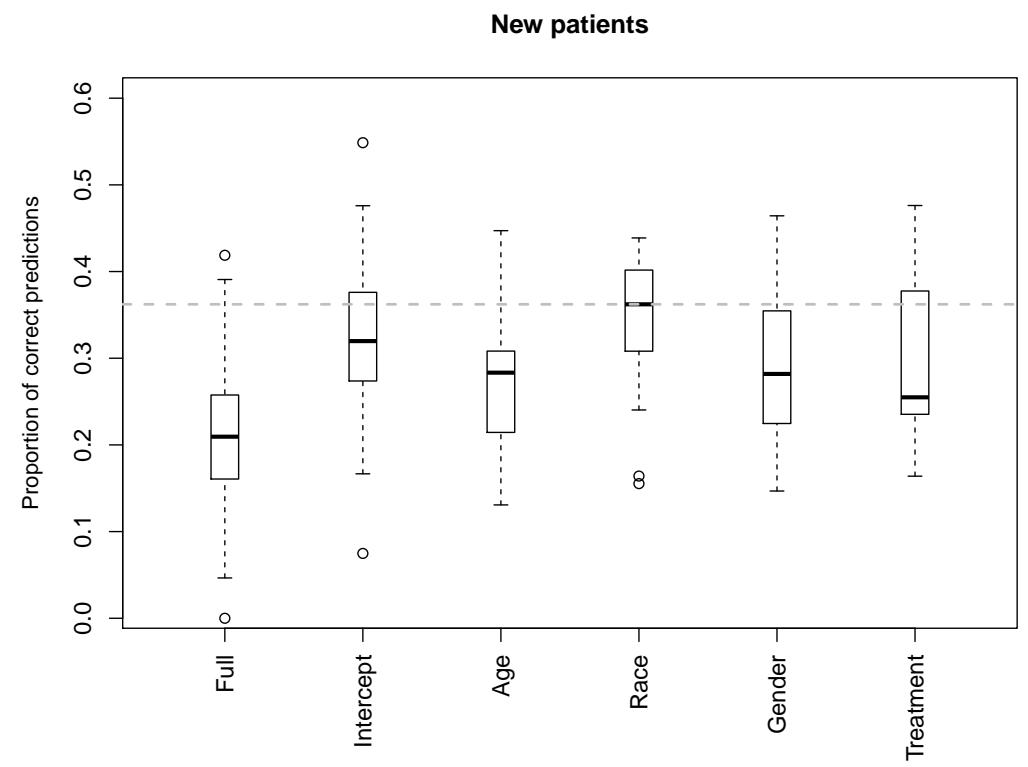

FIG 3. Simulation experiment for model selection using new patients. Each boxplot represents the performance of HARM with a subset of the available covariates. The model using just race covariates performed the best out of the models we considered. 


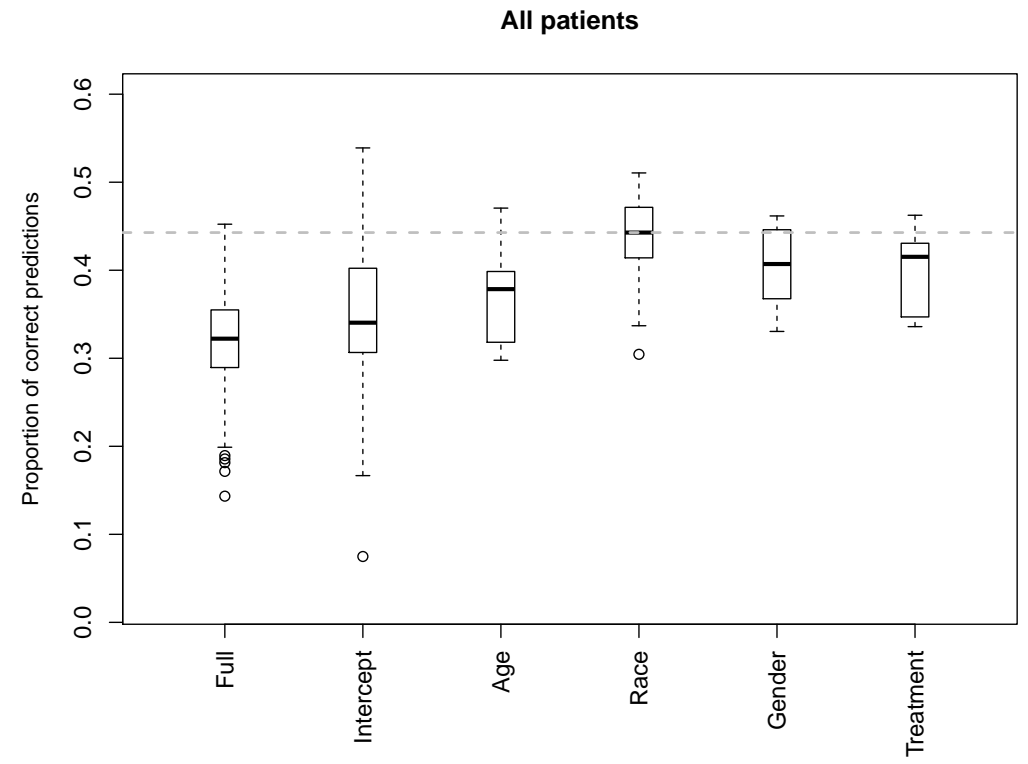

FIG 4. Simulation experiment for model selection using all patients (both partially observed and new patients). Each boxplot represents the performance of HARM with a subset of the available covariates. The model using just race covariates performed the best out of the models we considered. 


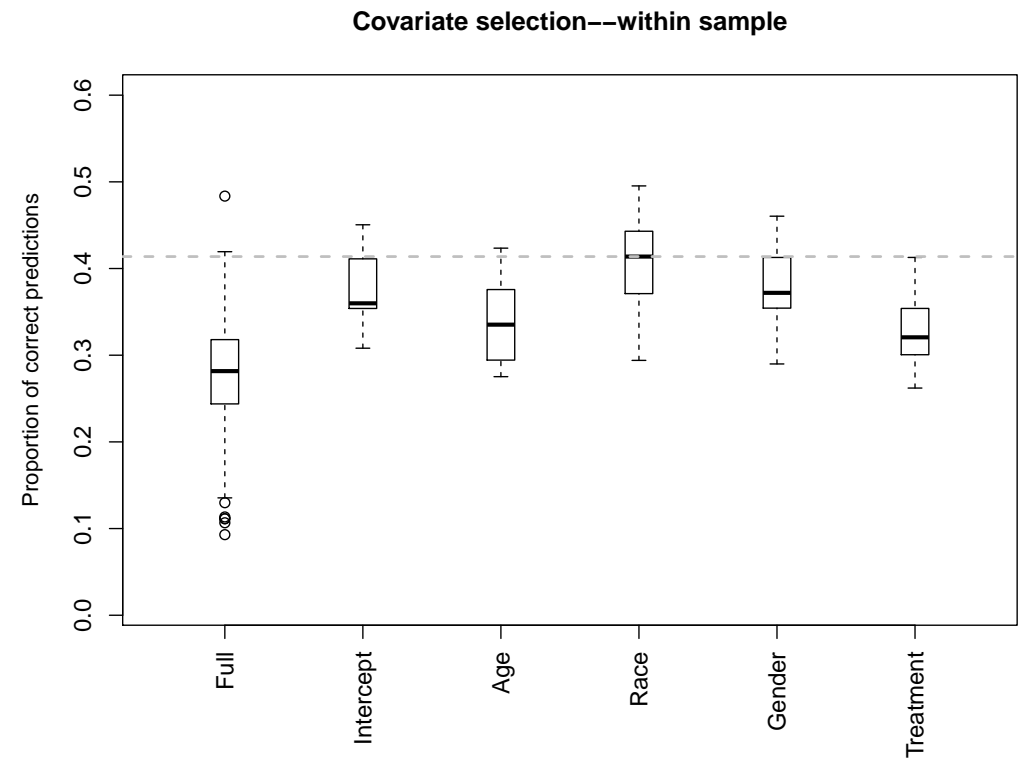

FIG 5. Simulation experiment for in-sample prediction. Each boxplot represents the performance of HARM for a specific with a subset of the available covariates. For each run of the simulation, patients we evaluated the performance of HARM in predicting the sequence of encounters used for training. 